\title{
Numerical Version of Self-Consistent Scheme for Heterogeneous Materials
}

\author{
Mengting $\mathrm{Xu}$, Limei $\mathrm{Xu}$ and Xiaomei Xie \\ School of Astronautics and Aeronautics, University of Electronic Science and Technology, Chengdu China
}

\begin{abstract}
The self-consistent scheme (Budiansky, 1965) is one of the analytical methods to predict the effective properties of heterogeneous material. However, it has been noticed that this scheme cannot take the micro-structural information into the estimation. The full scale numerical simulation considering elaborately the micro-morphological characteristics, under certain conditions, needs too much computational resources (memory and time). A numerical version of self-consistent scheme based on finite element analysis is proposed to overcome the above drawbacks by combining the characteristics of numerical simulation and self-consistent scheme. As a demonstration, such scheme is applied to estimate the effective mechanical properties of bi-continuous composite in which all the constituent phases are on the equal status. The prediction is in good agreement with the full scale numerical simulation while the requirement on computational resource is reduced dramatically.
\end{abstract}

Keywords: bi-continues composite, effective mechanical properties, self-consistent scheme, numerical simulation

\section{Introduction}

The research on the effective elastic properties of heterogeneous materials has been attracting great attention in the last few decades. Various analytical methods, which can be classified into "bound method" and "approximated method" have been proposed. VoigtReuss bounds [1] and Hashin-Shtrikman bounds [2] are the two prominent pairs of lower and upper bounds. Approximated method includes self-consistent scheme (Budiansky [3], Hill [4]), three-phase model (Christensen [5]) and Mori-Tanaka method ([6]). For the selfconsistent scheme, the essential point is to treat each constituent phase as an inhomogeneity embedded in a homogenized material with yet to be determined effective properties. In this context, each constituent phase is treated on the equal footing. And bi-continuous material is composed of multiple constituents which are on the equal status. It means the matrix and particulate cannot be clearly distinguished. Due to such micro-structural characteristics, the self-consistent scheme can be applicable for predicting the effective properties of the bicontinuous material. However, such scheme is not able to take the microstructural characteristics, such as the size, shape and distribution of its components, into the "over simplified" model.

With the progress of computational techniques and capabilities, numerical simulations, like finite element method, are widely employed to investigate the mechanical behavior and properties of heterogeneous material. A comprehensive discussion on the numerical simulation has been given by Cailletaud et al. [7] and Zohdi and Wriggers [8]. A full scale numerical simulation for the material's real microstructure has been considered as "exact" approach for the effective modulus prediction. However, it has been noticed that even with the most recent and ever improving development of computing and memory power, the real full scale numerical simulations are still limited if it is not impossible.

The aforementioned advantages and disadvantages of the pure analytical and the pure numerical approaches lead us to a possibility of mixing the analytical and the numerical schemes, namely, a numerical version of selfconsistent scheme. The objective of the numerical version of self-consistent scheme is, on the one hand, to be able to deal with the various micro-morphological information, such as size, shape and their distributions, in the heterogeneous material which cannot be considered by the analytical scheme. On the other hand, this scheme requires much less computational resources, like time and memory space. In a finite element analysis, this means the proposed scheme needs much less number of elements in comparison with the full scale numerical analysis.

This article will be organized as followings. Section 2 introduces the idea of numerical self-consistent scheme when predicting the effective properties of heterogeneous materials. Based on the proposed scheme, finite element simulation for certain bi-continuous material are conducted using a commercial finite element package (ANSYS) in Section 3. Conclusion is given in the last section. 


\section{Numerical self-consistent scheme for simulation models}

To illustrate the numerical self-consistent scheme, a twophase bi-continuous material in two-dimension is considered. The specimen for modeling the macroscopically isotropic and homogenous composite material is defined as $\mathrm{L} \times \mathrm{L}$, ' $\mathrm{L}$ ' is the macroscopic dimension of the composite material. The specimen is modeled by $N \times N$ domains with equal size $d \times d$ and each constituent phase is isotropic. The domain size ' $d$ ' is considered as a characteristic dimension with constant value of the microstructure. Following the finite element scheme described elaborately in our published paper [9], a realization of two-phase bi-continuous material used for the full scale numerical simulation is presented in Figure 1. The two phases are randomly distributed in the specimen according to their area fraction. It will be shown that reliable effective elastic modulus can only be achieved when domains reach a very large number.

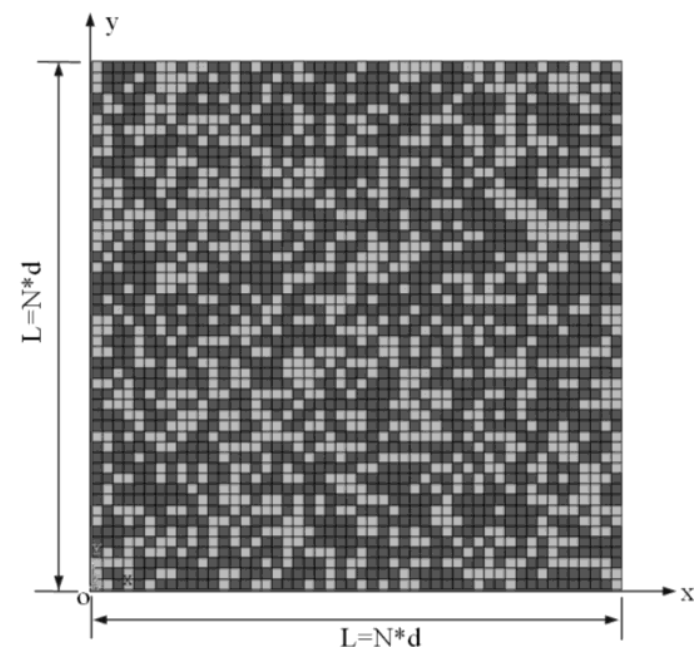

Figure 1. A realization of two-phase bi-continuous material. White color represents phase 1 with area fraction is $40 \%$ while black color denotes phase 2 with $60 \%$ area fraction. ' $N$ ' is the number of domains of each edge. ' $d$ ' is the characteristic size for the domain.

Incorporating the essential point of analytical selfconsistent scheme proposed by Budiansky, a finite element model, we name the numerical self-consistent model, is constructed. Figure 2 is a realization of the numerical self-consistent model corresponding to a twophase bi-continuous material. The core of the model is made of the same structure as the full-scale numerical model, while the outer material is "yet to be determined" effective medium. Since the effective medium has uniform elastic constants, the number of elements used in the effective region is much less than the "composite region". Therefore, the self-consistent scheme saves a substantial amount of the computational resources. At the meantime, such model captures the details of the microstructure of composite material. To evaluate the effective elastic constants, iterative procedure is employed following the framework of analytical self-consistent scheme. When the difference of the ith value and $(i+1)$ th prediction is within the given error, the $(i+1)$ th value is the effective Young's modulus.

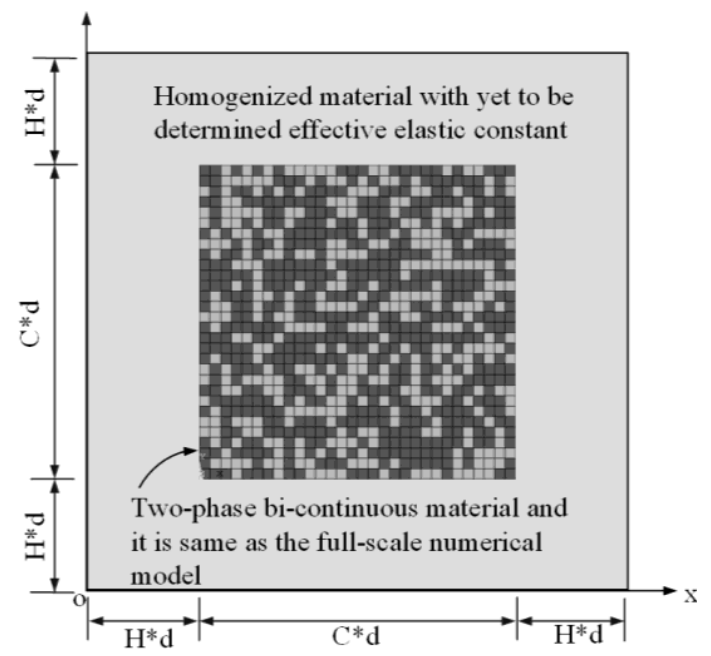

Figure 2. A realization of self-consistent model for the twophase bi-continuous material. The inside body is simulated in micro-structural level and embedded in the outside body which is homogenized with yet to be determined effective elastic constants. The inside body is composed of domains with area fraction $40 \%$ for phase 1 (white phase) and $60 \%$ for phase 2 (black phase). ' $\mathrm{C}$ ' is domains' number of the core structure's edge.

\section{Finite element simulation and discus- sion}

In order to avoid the numerical error due to the mismatch of the material properties between the adjacent domains, the process of mesh refinement is conducted during finite element simulation to relax the stress concentration and smooth the displacement variation between domains with different material properties. Besides, higher order element, namely twenty-node hexahedral element is adopted to improve the accuracy and accelerate the speed of convergence.

Furthermore, the concept of statistics is adopted due to the random distribution of two constituent phases over the established model. In the present work, the "population" refers to the specimen with a specific phase area fractions and material properties. For all the specimens in the population, the mean value of the effective modulus is denoted by $\mu$ and corresponding standard deviation is denoted by $\sigma$. A sample consisting of $n(=30)$ specimens is taken to infer the statistic data $(\mu$ and $\sigma$ ) of the population according to Central Limit Theorem (Mendenhall et al.[10]). Hence, a pair of statistical data, i.e., the sample mean value $(\bar{X})$ and the sample standard deviation (s), is determined. And, the mean value $(\mu)$ of population (the specimen including certain number of the grains) can be inferred within a $95 \%$-confidence interval by

$$
\bar{X}-1.96 \frac{s}{\sqrt{n}} \leq \mu \leq \bar{X}+1.96 \frac{s}{\sqrt{n}}
$$




\section{ICIEA 2016}

In evaluating the effective elastic Young's modulus of the selected bi-continuous material, the following loading and boundary conditions are imposed.

where $a$ is prescribed displacement, $u_{x}$ and $u_{y}$ represent the displacement along the direction $x$ and $y$ in the global coordinate system.

$$
\begin{cases}u_{x}=0 & \text { at } x=0 \\ u_{x}=a & \text { at } x=L \\ u_{y}=0 & \text { along } x=0, y=0\end{cases}
$$

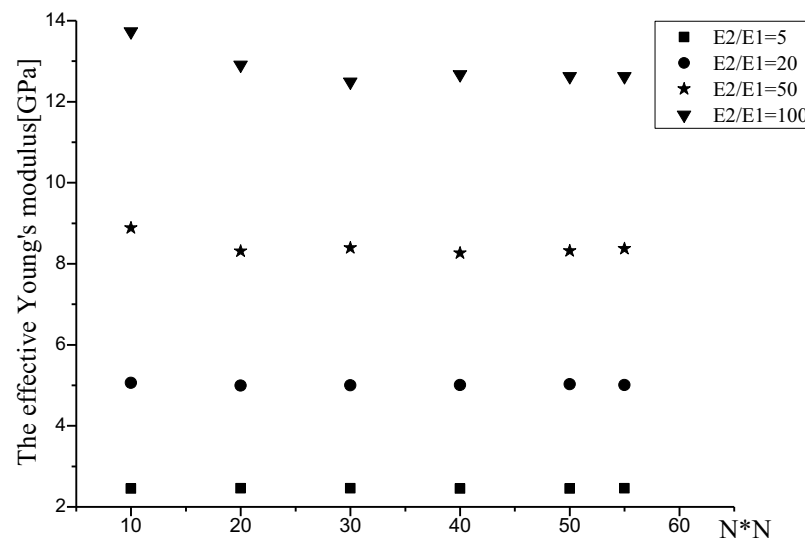

Figure 3. The effective Young's modulus in terms of the size of the specimen with different ratio of Young's modulus of two constituent materials.

Fig. 3 presents the effective Young's modulus obtained from different models with various Young's modulus ratio of two constituent materials, namely E2/E1 $=5,20,50,100$, respectively, in terms of the size of the specimen. It is noted, with the increase of the specimen's size, the effective Young's modulus tends to a stable value, and then the size of Representative Volume Element (RVE) for each material can be determined. Table 1 lists the size of RVE for materials with different E2 / E1. It is seen the size of RVE is larger when the modulus ratio is bigger. As shown, to obtain the effective Young's modulus for material with $E 2 / E 1=100$, the corresponding RVE should have $50 \times 50$ domains.

Table 1. The RVE for materials with different E2 / E1

\begin{tabular}{|c|c|c|c|c|}
\hline E2/E1 & 5 & 20 & 50 & 100 \\
\hline Size of RVE(N*N) & $20 * 20$ & $40 * 40$ & $50 * 50$ & $50 * 50$ \\
\hline
\end{tabular}

With reference to Fig. 2, the dimension of ' $H$ ' is a critical size when establishing the corresponding selfconsistent model. To determine the size ' $H$ ', the twophase bi-continuous material with $E 2 / E 1=20$ and 100 is taken into account, respectively. The corresponding specimen size is assumed to be identical with the predicted RVE size, namely $40 \times 40$ for $E 2 / E 1=20$, $50 \times 50$ for $E 2 / E 1=100$. In the process of estimation, the initial Young's modulus and Poisson's ratio are assumed to be the average value of the mechanical properties of the constituent phase. The obtained effective Young's modulus is depicted with the variation of ' $H$ ' and iterative time in Fig. 4. The number of element and node in the finite element models and computational time for different models are summarized in Table 2.

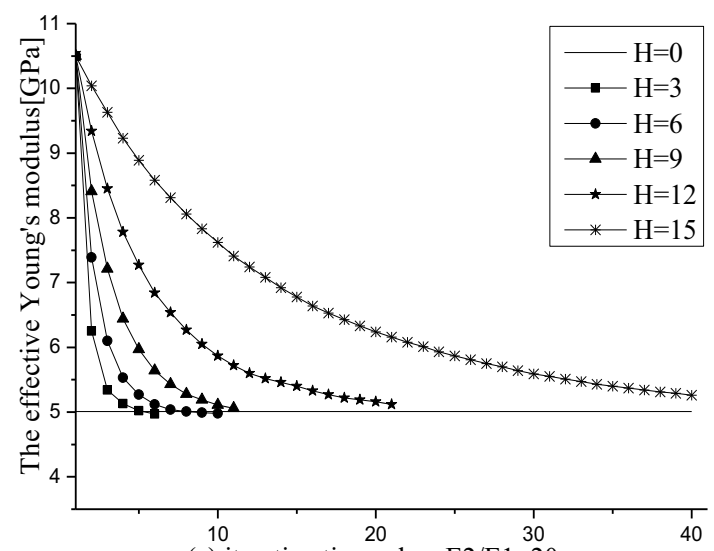

(a) iterative time when $\mathrm{E} 2 / \mathrm{E} 1=20$

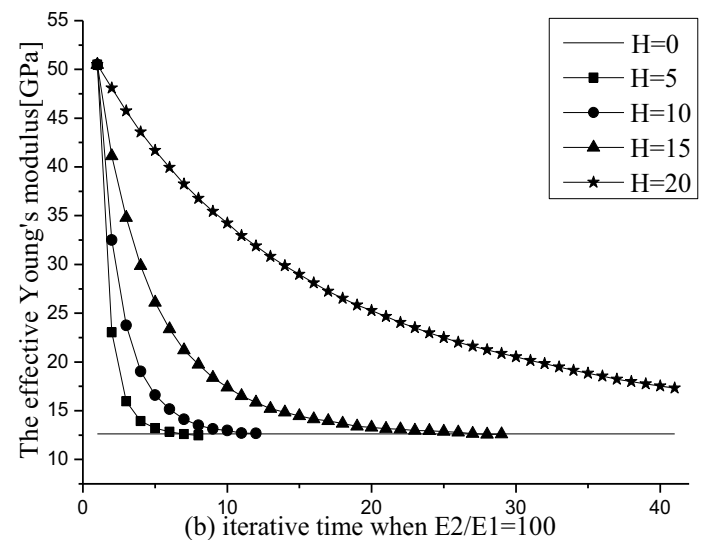

Figure 4. The effective Young's modulus of the composite material with changing ' $H$ ', in terms of iteration number. (a) $E 2$ $/ E 1=20$, (b) $E 2 / E 1=100$

Table 2. The finite element models and computational time for different models while the specimen size is $50 \times 50$ and $E 2 / E 1=100$.

\begin{tabular}{|c|c|c|c|c|c|c|}
\hline The size of ' $H$ ' & $\begin{array}{c}\text { The effective } \\
\text { Young's modulus } \\
{[\mathrm{GPa}]}\end{array}$ & $\begin{array}{c}\text { Element number } \\
{[\text { Ten thousand] }}\end{array}$ & $\begin{array}{c}\text { Node number } \\
{[\text { Ten thousand }]}\end{array}$ & $\begin{array}{c}\text { CPU time for an } \\
\text { iteration[s] }\end{array}$ & $\begin{array}{c}\text { Number of } \\
\text { iteration }\end{array}$ & $\begin{array}{c}\text { Total time } \\
{[\mathrm{s}]}\end{array}$ \\
\hline 0 (full-scale) & 12.62 & 16 & 48.1601 & 62.853 & 1 & 62.853 \\
\hline 5 & 12.46 & 15.84 & 47.6641 & 68.516 & 8 & 548.1 \\
\hline 10 & 12.68 & 9.45 & 28.4581 & 47.315 & 11 & 520.5 \\
\hline 15 & 12.60 & 4.68 & 14.1121 & 23.026 & 22 & 506.6 \\
\hline 20 & 17.31 & 1.53 & 4.6261 & 7.675 & 46 & 353.1 \\
\hline
\end{tabular}


As indicated in Figure 4, the effective Young's modulus will converge to the value obtained from the full scale model $(\mathrm{H}=0)$, no matter what the size of the $\mathrm{H}$ is, with the increase of the iteration. However, the speed of convergence becomes more and more slow as the size of ' $H$ ' increases.

Reference to the data in Table 2, it is seen that the self-consistent scheme models need less number of elements and nodes in compared with the full-scale numerical analysis. When ' $H$ ' is 15 , the number of elements and nodes in full-scale model is about 4 times of those in the self-consistent scheme model.

As for the computational time, the full-scale model expends almost 3 times in one iteration more than the self-consistent models while the total time is much more for the self-consistent models. In this context, the proposed self-consistent model reduces the requirement of computational resource at the expense of computational time. Considering both calculating time and the convergence rate of the effective Young's modulus, the selected ' $H$ ' and RVE size denoted by ' $\mathrm{N}$ ' are summarized in Table 3, for different two-phase bicontinuous materials with various $E 2 / E 1$.

Table 3. Suitable H and N for different ratio of Young's modulus of constituent phase in two-phase bi-continuous material

\begin{tabular}{|c|c|c|c|c|}
\hline $\mathrm{E} 2 / \mathrm{E} 1$ & 5 & 20 & 50 & 100 \\
\hline $\mathrm{H} / \mathrm{N}$ & $0 / 20$ & $6 / 40$ & $10 / 50$ & $15 / 50$ \\
\hline
\end{tabular}

\section{Conclusions}

To predict the effective properties of heterogeneous material, a numerical self-consistent model is proposed based on finite element method. This model consider the real microstructural characteristics as the full-scale numerical model in the core, and the outer region is "yet to be determined" effective medium with uniform elastic constants. Such model overcome the shortcoming of the analytical version of self-consistent scheme by incorporating the interaction among the material's constituent phases. In comparison with the full-scale numerical model, the proposed numerical self-consistent method has less requirement on the computational resources, such as time and memory space. Thus, in the case of limited computational resource, the proposed numerical self-consistent scheme is effective in studying the effective properties of composites, especially for the materials which constituent phases have high contrast in their properties.

\section{References}

1. R. Hill, Proc. Phys. Soc. A65 (1952) 349-354.

2. Z. Hashin, S. Shtrikman, J. Mech. Phys. Solid 10 (1962) 335-342.

3. B. Budiansky, J. Mech. Phys. Solids. 13 (1965) 223227.

4. R. Hill, J. Mech. Phys. Solids. 13 (1965) 213-222.

5. R. M. Christensen, K. H. Lo, J. Mech. Phys. Solids 27 (1979) 315-330.

6. T. Mori, K. Tanaka, Acta Metall. 21 (1973) 571-574.

7. G. Cailletaud, S. Forest, D. Jeulin, F. Feyel, I. Galliet, V. Mounoury, S. Quilici. Computational Materials Science. 27 (2003) 351-374

8. T. I. Zohdi, P. Wriggers, An introduction to computational micromechanics. Second edition. Springer-Verlag Berlin and Heidelberg $\mathrm{GmbH}$ Publishing (2008).

9. X. M. Xie, H. Fan, Materials Science and Engineering A. 527 (2010) 5452-5461.

10. W. Mendenhall, R. J. Beaver, B. M. Beaver, Introduction to Probability and Statistics, 12th edition, Thomson Learning Publishers, Singapore (2006). 